\title{
Damage Identification of Wind Turbine Blades Using Piezoelectric Transducers
}

\author{
Seong-Won Choi, ${ }^{1}$ Kevin M. Farinholt, ${ }^{2}$ Stuart G. Taylor, ${ }^{2}$ \\ Abraham Light-Marquez, ${ }^{2}$ and Gyuhae Park ${ }^{1,2}$ \\ ${ }^{1}$ School of Mechanical Systems Engineering, Chonnam National University, Gwangju 500-757, Republic of Korea \\ ${ }^{2}$ Engineering Institute, MS T001, Los Alamos National Laboratory, Los Alamos, NM 87545, USA
}

Correspondence should be addressed to Gyuhae Park; gpark@chonnam.ac.kr

Received 14 February 2013; Accepted 14 June 2013; Published 7 April 2014

Academic Editor: Jung-Ryul Lee

\begin{abstract}
Copyright (C) 2014 Seong-Won Choi et al. This is an open access article distributed under the Creative Commons Attribution License, which permits unrestricted use, distribution, and reproduction in any medium, provided the original work is properly cited.

This paper presents the experimental results of active-sensing structural health monitoring (SHM) techniques, which utilize piezoelectric transducers as sensors and actuators, for determining the structural integrity of wind turbine blades. Specifically, Lamb wave propagations and frequency response functions at high frequency ranges are used to estimate the condition of wind turbine blades. For experiments, a $1 \mathrm{~m}$ section of a CX-100 blade is used. The goal of this study is to assess and compare the performance of each method in identifying incipient damage with a consideration given to field deployability. Overall, these methods yielded a sufficient damage detection capability to warrant further investigation. This paper also summarizes the SHM results of a full-scale fatigue test of a $9 \mathrm{~m} \mathrm{CX}-100$ blade using piezoelectric active sensors. This paper outlines considerations needed to design such SHM systems, experimental procedures and results, and additional issues that can be used as guidelines for future investigations.
\end{abstract}

\section{Introduction}

Wind turbines are becoming a larger source of renewable energy in the world. The US government projects that $20 \%$ of the US electrical supply could be produced via wind power by 2030 [1]. To achieve this goal, the turbine manufacturers have been increasing the size of the turbine blades, often made of composite materials, to maximize power output. As a result of severe wind loadings and the material level flaws in composite structures, blade failure has been a more common occurrence in the wind industry. Monitoring the structural health of the turbine blades is particularly important as they account for $15-20 \%$ of the total turbine cost. In addition, blade damage is the most expensive type of damage to repair and can cause serious secondary damage to the wind turbine system due to rotating imbalance created during blade failure. Therefore, it is imperative that a structural health monitoring (SHM) system be incorporated into the design of the wind turbines in order to monitor flaws before they lead to a catastrophic failure.
There has been a considerable research effort focused on applying SHM techniques on wind turbine blades [2, 3]. However, most of these studies focus on a single technique for damage detection; consequently very little work has been done to compare the results of multiple active-sensing techniques. Thus, the goal of this study is to assess the relative performance of high-frequency SHM techniques, namely, Lamb wave propagation and frequency response functions (FRFs), as a way to nondestructively monitor the health of a wind turbine blade with piezoelectric active sensors. In conjunction, consideration is given to employing multiple techniques together as a means of increasing the effectiveness of SHM for detecting and locating damage. This combination method is possible because of the multifunctional nature of the piezoelectric active sensors. In this paper, an array of piezoelectric sensors on a $1 \mathrm{~m}$ section of a $9 \mathrm{~m} \mathrm{CX}-100$ blade is used for simulated damage detection under the laboratory setting. Once the damage detection performance was characterized, the piezoelectric active-sensing techniques are applied to SHM of a full-scale $9 \mathrm{~m} \mathrm{CX}-100$ blade, where the 
blade was dynamically loaded in a fatigue test until reaching catastrophic failure.

\section{SHM Using Piezoelectric Active Sensors}

Piezoelectric transducers have been widely used in SHM applications because they provide both sensing and actuation capabilities within a local area of the structure. These transducers could easily provide high-frequency excitations, and the subsequent structural responses could be captured by the same excitation sources. Two active and local sensing techniques, including Lamb wave propagations and FRF at high frequency ranges, are briefly summarized in this section.

2.1. Lamb Wave Propagations. Since the 1960s, the ultrasonic research community has studied Lamb waves for the nondestructive evaluation of plates [4]. Lamb waves are mechanical waves corresponding to vibration modes of plates with a thickness in the same order of magnitude as their wavelength. The advances in sensor and hardware technologies for efficient generation and detection of Lamb waves and the need to detect subsurface damage in laminate composite structures have led to a significant increase in the use of Lamb waves for detecting defects in structures.

The dispersive nature of Lamb waves means that the different frequency components of the Lamb waves travel at different speeds and that the shape of the wave packet changes as it propagates through solid media. There are two types of modes that form in a plate when excited with Lamb waves: asymmetric $(A)$ and symmetric $(S)$. The asymmetrical modes are analogous to shear waves (equivalent to $S$ waves in earthquake engineering), while symmetrical modes are analogous to compression waves (equivalent to $P$ waves in earthquake engineering). The selection of the excitation frequency for Lamb waves must be made so as to excite a structure at a certain mode $\left(S_{0}\right.$ or $\left.A_{0}\right)$ and to avoid any higher modes that might also be present. Lamb wave propagation methods look for the possibility of damage by tracking changes in transmission velocity and wave attenuation/reflections. Several methods have been proposed to enhance the interpretation of the measured Lamb wave signals to detect and locate structural damage. They are based on changes in wave attenuations using wavelets [5], timefrequency analysis [6], wave reflections [7], and time of flight information [8]. A more complete description on the Lamb wave propagation technique can be found in [9].

\section{Frequency Response Functions}

The basic concept of high-frequency response functions is to use high frequency vibrations to monitor local regions of a structure for changes in the structure's parameters. It is a well-known fact that FRFs represent a unique dynamic characteristic of a structure. From the standpoint of SHM, damage will alter the stiffness, mass, or energy dissipation properties of a system, which, in turn, results in the changes in the FRF of the system [10]. Several investigations have been made to utilize the measured FRF for detecting damage in

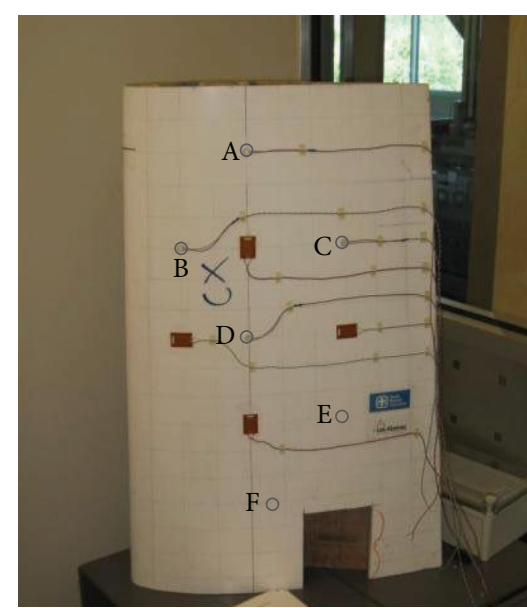

FIGURE 1: Turbine blade section.

structures [11-13]. In addition, the piezoelectric impedancebased method [14] is also in line with those based on FRF, because it indirectly measures the mechanical impedance of a structure over select frequency ranges. By utilizing piezoelectric active sensors, the FRF could be measured up to hundreds of $\mathrm{kHz}$ ranges, which allows the method to be sensitive to small defects in the structure and not sensitive to operational or boundary condition changes, which occurs at relatively low frequency ranges.

\section{Experimental Procedure and Results}

The experimental setup under a laboratory setting consisted of a $1 \mathrm{~m}$ long $\times 0.55 \mathrm{~m}$ wide section of a CX-100 wind turbine blade with a thickness that varies from $0.3 \mathrm{~cm}$ (trailing edge) to $10 \mathrm{~cm}$ (spar cap) instrumented with six piezoelectric patches, labeled A-F, as shown in Figure 1. Figure 1 also shows 4 rectangular macrofiber composite (MFC) sensors attached to the turbine blade; these sensors were not used in this study. The patches $\mathrm{E}$ and $\mathrm{F}$ are boned inside the blade. The CX-100 is a carbon reinforced composite $9 \mathrm{~m}$ turbine blade designed by Sandia National Laboratory (SNL) [15]. The piezoelectric patches are $13 \mathrm{~mm}$ in diameter and are attached to the surface of the turbine blade using cyanoacrylate adhesive. The use of 6 piezoelectric patches results in 15 possible wave propagation paths, labeled with the actuator patch first and the sensor patch second. The length of AF is measured at $600 \mathrm{~mm}$ as the longest path, and EF is the shortest distance at $220 \mathrm{~mm}$. The vertical paths (AD and $\mathrm{AE}$ ) are located directly on the center support spar of the blade, as seen in the cross section picture in Figure 2. The patches were connected to a National Instruments (NI) PXI data acquisition system for data acquisition.

4.1. Lamb Wave Propagations. The Lamb wave propagation experiments utilize the pitch-catch approach, where one of the piezoelectric patches is used as an actuator and another as a sensor. The actuator transmits a signal that travels, along the surface of the structure, to the sensor where it is recorded. 


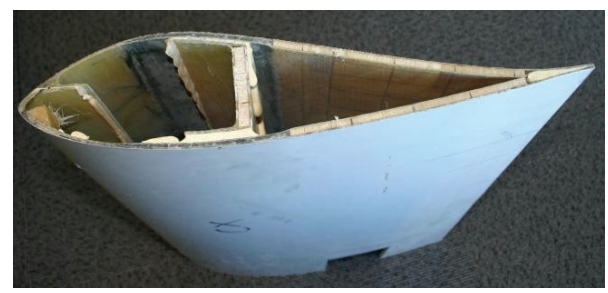

FIgure 2: Cross section view of CX-100.

To maximize the effectiveness of the Lamb wave technique the excitation frequency needs to be carefully selected. In order for damage detection to be possible the amplitude of the response must have a higher signal to noise ratio, and the response must be separated by a sufficient amount from the electromagnetic interference (EMI) to allow for proper identification of the arrival waveform. Due to the complexity of the blade section, traditional methods of predicting the ideal excitation frequency for homogeneous material [16] are not applicable and the ideal frequency was determined experimentally.

Overall, most of the paths showed desirable responses with an input frequency of $25 \mathrm{kHz}$ : three paths $(\mathrm{AD}, \mathrm{BD}$, and $\mathrm{DF}$ ) gave more advantageous responses with an input frequency of $200 \mathrm{kHz}$ and three paths (AE, AF, and BE) did not provide an acceptable response at any frequency leaving the 12 paths. It also has identified that the travel distance of the waves is about $50 \mathrm{~cm}$; thus for monitoring of the blade using Lamb wave propagations, one may need to either deploy a large number of traditional sensors or design an ultrasonic transducer to more effectively excite and sense a certain frequency range for SHM.

After the excitation frequency has been determined, multiple baselines for the undamaged structure were recorded. These baselines contain variations in the boundary conditions to attempt to simulate potential real world variability, including mass loading and manually induced vibrations to the structure. Damage was then introduced by applying a piece of industrial putty to the surface of the turbine blade in the path of propagation of the Lamb waves. The putty serves to change the damping properties of the structure in a localized area, similar to the effects of a delamination. The putty was approximately $5 \mathrm{~cm} \times 5 \mathrm{~cm}$, with $0.5 \mathrm{~cm}$ thickness. An example of recorded signal is shown in Figure 3. The responses (path BC) are recorded in the direct path before and after the simulate damage was applied. One can clearly see the attenuation of the first arrival wave caused by the simulated damage.

The signal processing technique used was based on monitoring the changes in the energy content of the propagated waves. First, the arrival signals were captured in time to avoid interference with boundary reflected waves. This captured signal was then converted into the frequency domain using a discrete Fourier transform. These frequency domain data were integrated, using the trapezoidal approximation method in an attempt to determine the energy content of the recorded response. A damage index was then created, which is defined as the percent difference between the true baseline and each of the other recorded values. To incorporate all of the baseline readings into a single value, all of the damage indices for the baseline cases were averaged and then compared with the correlation coefficient for the damaged case. The result of the induced damage (shown in Figure 3 ) is illustrated in Figure 4. Ten baselines were first measured to construct the baseline database under different boundary conditions. The average of all the baselines is taken as an undamaged value and compared against the damage index for the damaged case.

The result shows that path $\mathrm{BC}$ indicates very clear signs of damage. In addition, pathes $\mathrm{AC}$ and $\mathrm{CE}$ is also affected by this damage. Therefore, it is possible to get the approximate location of damage based on the paths. AC and CD both have very similar magnitude damage indices which would suggest that the damage is located about the same distance from those 2 paths, which also agrees with the indication that the damage was on or near the $\mathrm{BC}$ path. Based on the fact that the $\mathrm{AB}$ and $\mathrm{BD}$ paths are not indicating damage, it is likely that the damage is closer to the $\mathrm{C}$ piezoelectric patch. This process could approximately locate induced structural damage.

In most cases, this method could detect the simulated damage in the test, but only when damage was introduced close to the sensor-actuator paths. This low spatial detectability results from the relatively high damping present in composite structures, which limits the distance the Lamb wave can travel. This suggests that a large number of sensors would be needed to monitor the entire turbine blade. Furthermore, with the presence of the spar inside of the blade, the selection of the wave frequency is not always straightforward; piezoelectric transducers should be installed in such a way that they can avoid wave scattering caused by the spar.

4.2. High-Frequency FRF. Initially, the testing was conducted using an input frequency bandwidth of $30-80 \mathrm{kHz}$ on each sensor-actuator combination. The experimental results, where the same damage as in the previous section in Figure 4 was introduced, are shown in Figure 5. The high-frequency response displays the changes in shape due to the simulated damage condition. The top portion of the figure is the real part, while the bottom portion is the imaginary part of FRF. As can be seen in the figure, the real portion of the response shows more variability due to baseline changes, while the imaginary portion is very stable, which is the ideal behavior. Changes due to boundary conditions are slight shifts in the magnitude of the FRF at the resonance and antiresonance peaks. The changes caused by damage result in a complete change in the frequency response function.

Damage index was obtained using a correlation function between baselines and a new set of data. The correlation coefficient determines the linear relationship between the two data sets:

$$
\begin{aligned}
\rho= & \frac{1}{n-1} \\
& \times \frac{\sum_{i=1}^{n}\left(\operatorname{Im}\left(Z_{i, 1}\right)-\operatorname{Im}\left(\bar{Z}_{1}\right)\right)\left(\operatorname{Im}\left(Z_{i, 2}\right)-\operatorname{Im}\left(\bar{Z}_{2}\right)\right)}{\sigma_{z_{1}} \sigma_{z_{2}}},
\end{aligned}
$$



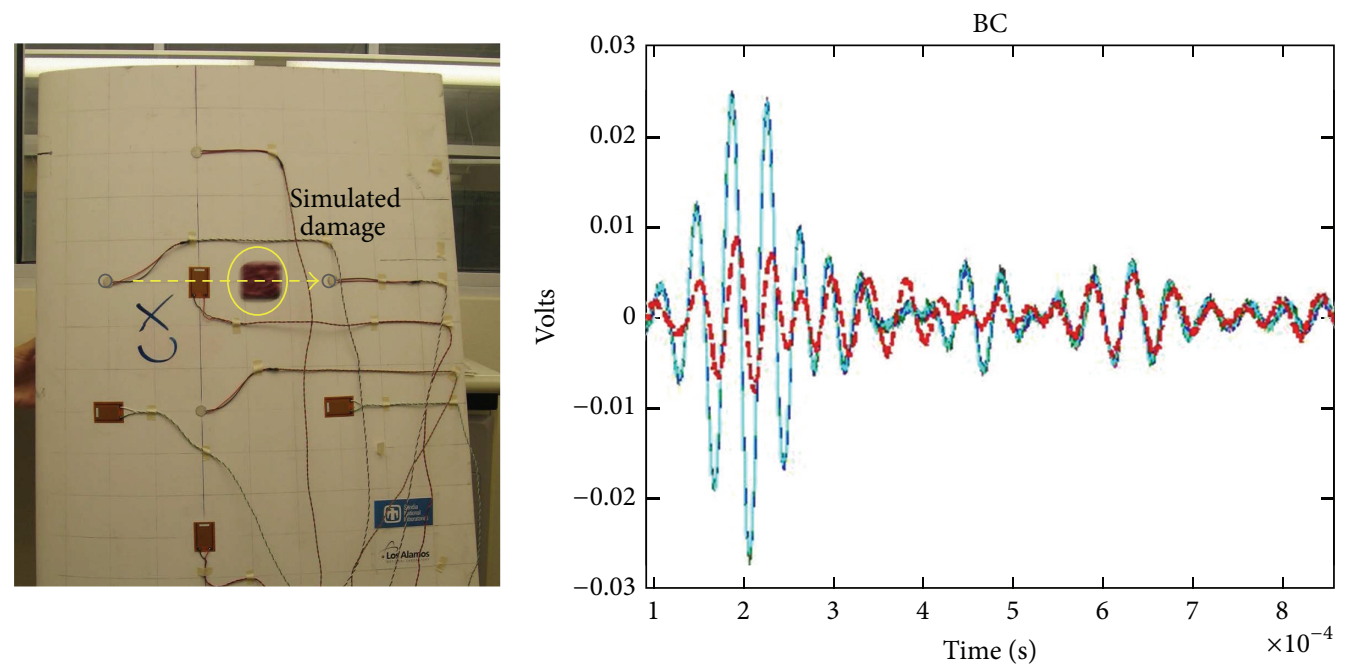

FIGURE 3: Turbine blade section with simulated damage and ten recorded baseline signals and damaged signal for path BC.

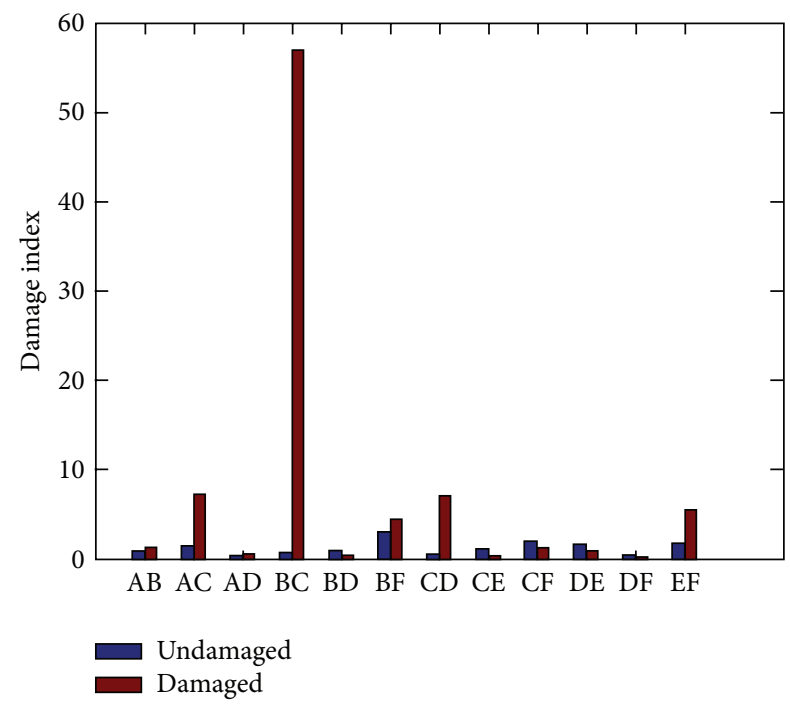

FIGURE 4: Damage index values for undamaged and damaged blade.

where $\rho$ is the correlation coefficient, $Z_{i, 1}$ is the baseline FRF data and $Z_{i, 2}$ is the compared FRF data at frequency $i, \bar{Z}_{1}$ and $\bar{Z}_{2}$ are the means of the signals, and the $\sigma$ terms are the standard deviations. For convenience, the feature examined in this study is $(1-\rho)$, in order to ensure that, with increasing damage or change in structural integrity, the metric values also increase. A greater damage metric value means that a certain degree of dissimilarity, with respect to a baseline measurement, is present in a particular measurement. The goal here is to show that this dissimilarity is directly related to the amount of damage present.

A correlation-based damage metric chart is illustrated in Figure 6 . The figure shows that almost every path is indicating the presence of damage on the structure. The only exception is the EF path, which is the farthest path from the damage location. Paths BC and AE have the largest damage indices
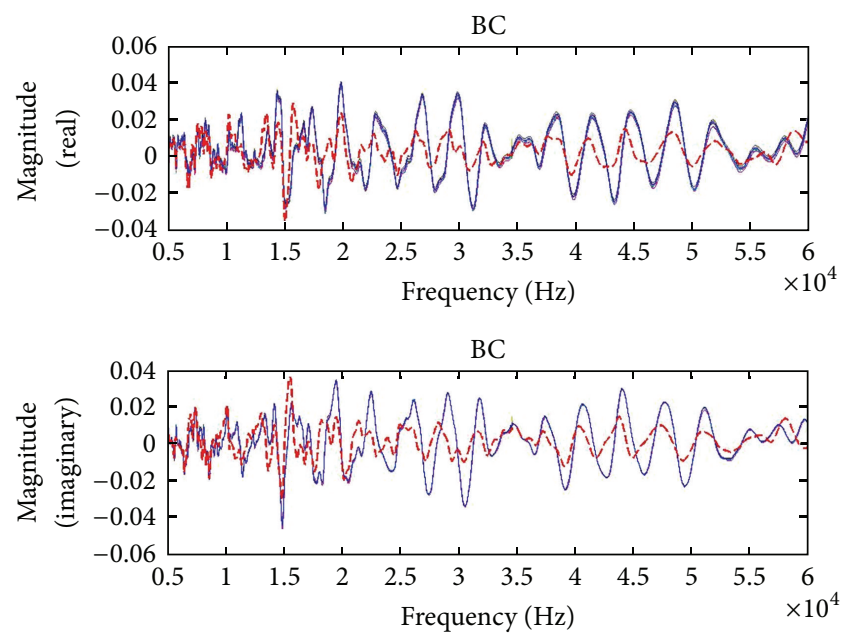

FIGURE 5: 15 recorded baseline signals and damaged signal for path BC.

(as a reminder the $\mathrm{AE}$ path did not provide an acceptable response for the Lamb wave method). This indicates that the damage is located on or very near to both of these paths.

Although extensive averaging was required to enhance SNR, this method could detect any damaged condition imposed into the blade. With the high-frequency range interrogated and relative high damping present in the structure, the damage localization was also observed; that is, more pronounced response changes if damage was introduced close to the transducers.

For the frequency response function method, the longer propagation paths were capable of producing an acceptable recorded response for damage detection compared to Lamb wave propagation. This suggests that it might be possible to limit the number of sensors required to cover the entire turbine blade. This is further aided by the fact that the FRF method was determined to have global damage detection 


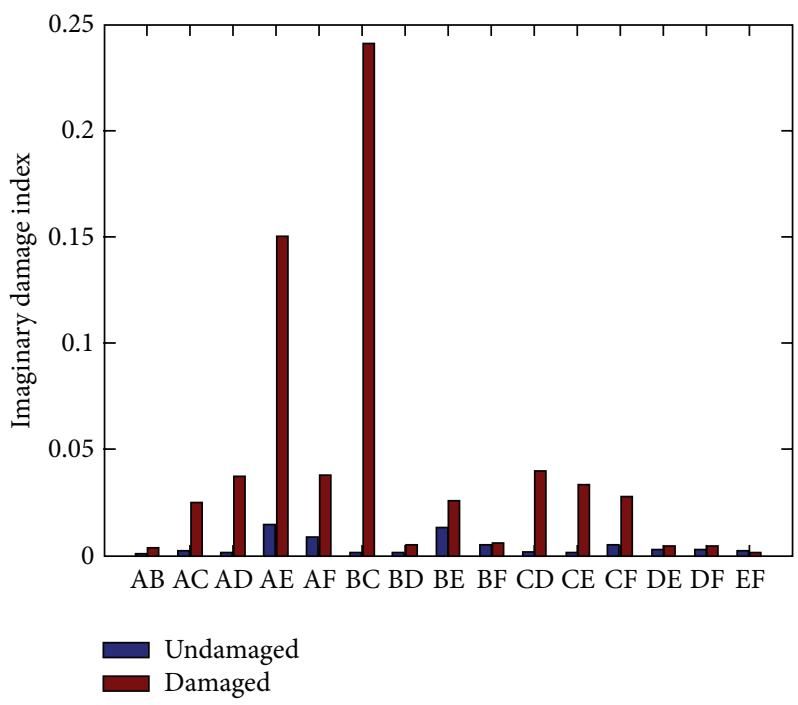

FIGURE 6: Damage index values using FRFs.

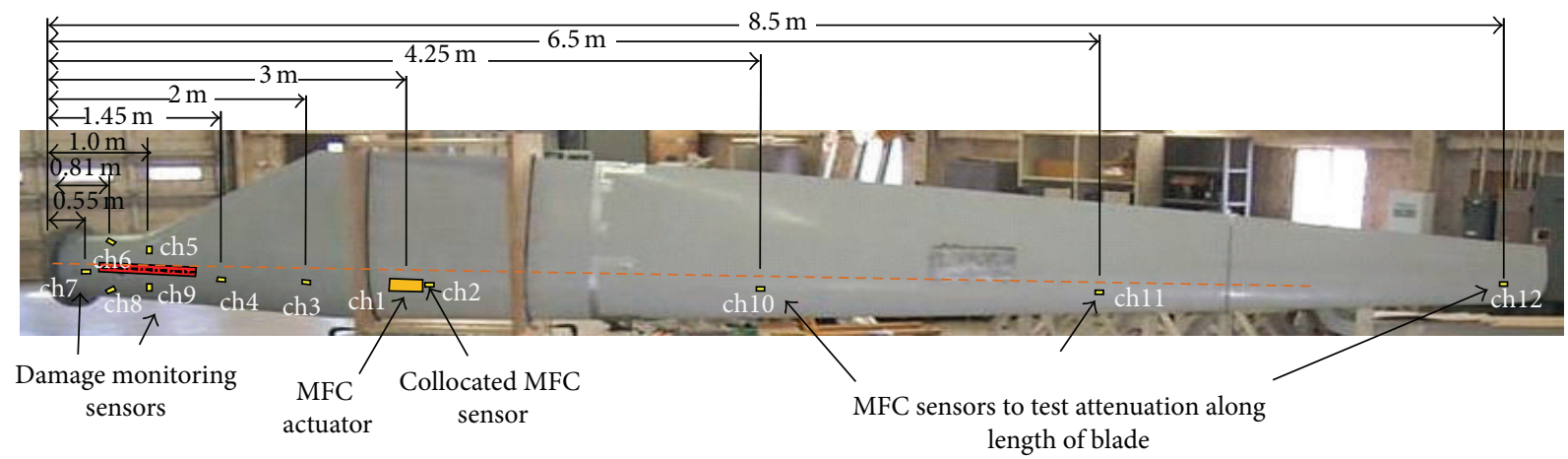

Figure 7: Overview of the fatigue test setup. A single MFC actuator in Ch1 is used to excite the blade, and 11 MFC sensors are used to measure the signal.

capability. In addition, it was discovered that the magnitude of the damage index is directly related to the proximity of the propagation path to the damage location. This feature can be utilized to determine the location of the damage with an acceptable degree of accuracy. Similar results were obtained for damage placed at multiple locations on the turbine blade. To summarize, the FRF method is an acceptable structural health monitoring technique for reliably detecting and locating damage in wind turbine blades and has the potential for limiting the number of sensors required to cover the entire structure.

4.3. Comparison of the Two Methods. The performances of two methods for SHM of wind turbine blades were investigated in this section. It was found that both methods were capable of detecting damage, which was simulated by placing a piece of industrial putty on the surface of the $1 \mathrm{~m}$ CX-100 blade section. Lamb wave techniques offer the ability to detect damage on or near the path of the propagating wave and can be used to approximately locate the damage. However, long paths (longer than $50 \mathrm{~cm}$ ) were incapable of transmitting a waveform along the entire path, due to the high damping properties of composite turbine blades. The frequency response method showed a greater ability to detect damage on a global scale and the proximity of the damage location to the magnitude of the damage index allowed for the location of the damage to be determined. The frequency response method was also capable of transmitting a meaningful signal for all the propagation paths, including the longer distances. Consequently, the FRF method would require fewer sensors mounted on the structure in order to detect the presence of damage. Additionally, the FRF method utilizes a random excitation over a given bandwidth for all the propagation paths, eliminating the need to find the ideal excitation frequency, as is required for the Lamb wave method. However, as this method uses standing waves, the performance in localization of the damage is poorer than the wave propagation approach.

\section{CX-100 Full-Scale Fatigue Testing}

A full-scale fatigue test of a CX-100 wind turbine blade was also performed by SNL at the National Renewable Energy Laboratory (NREL). An overview photograph of the 


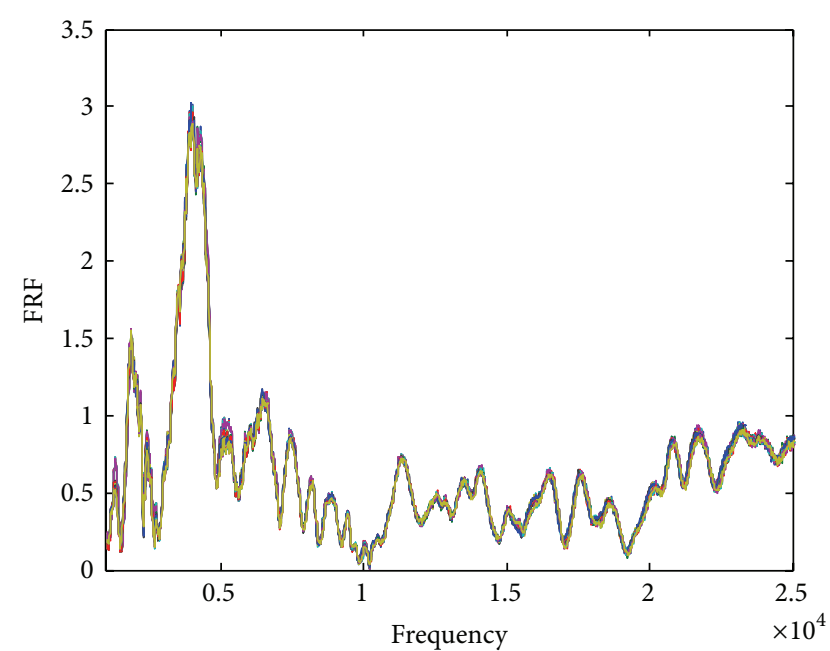

(a)

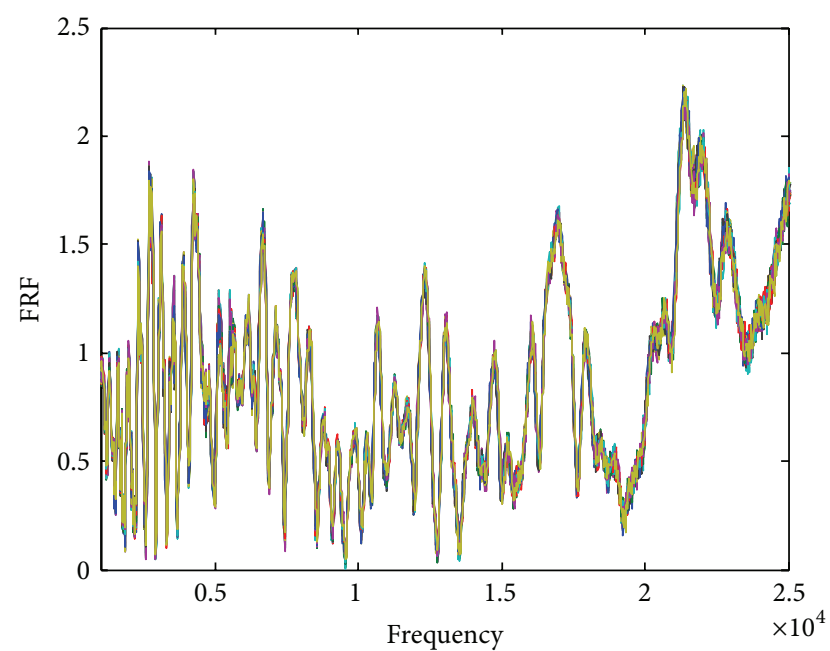

(b)

Figure 8: Identified FRFs. (a) Ch3, (b) Ch4. 20 measurements (from $260 \mathrm{k}$ to $506 \mathrm{k}$ cycles) are overlapped in the figure.
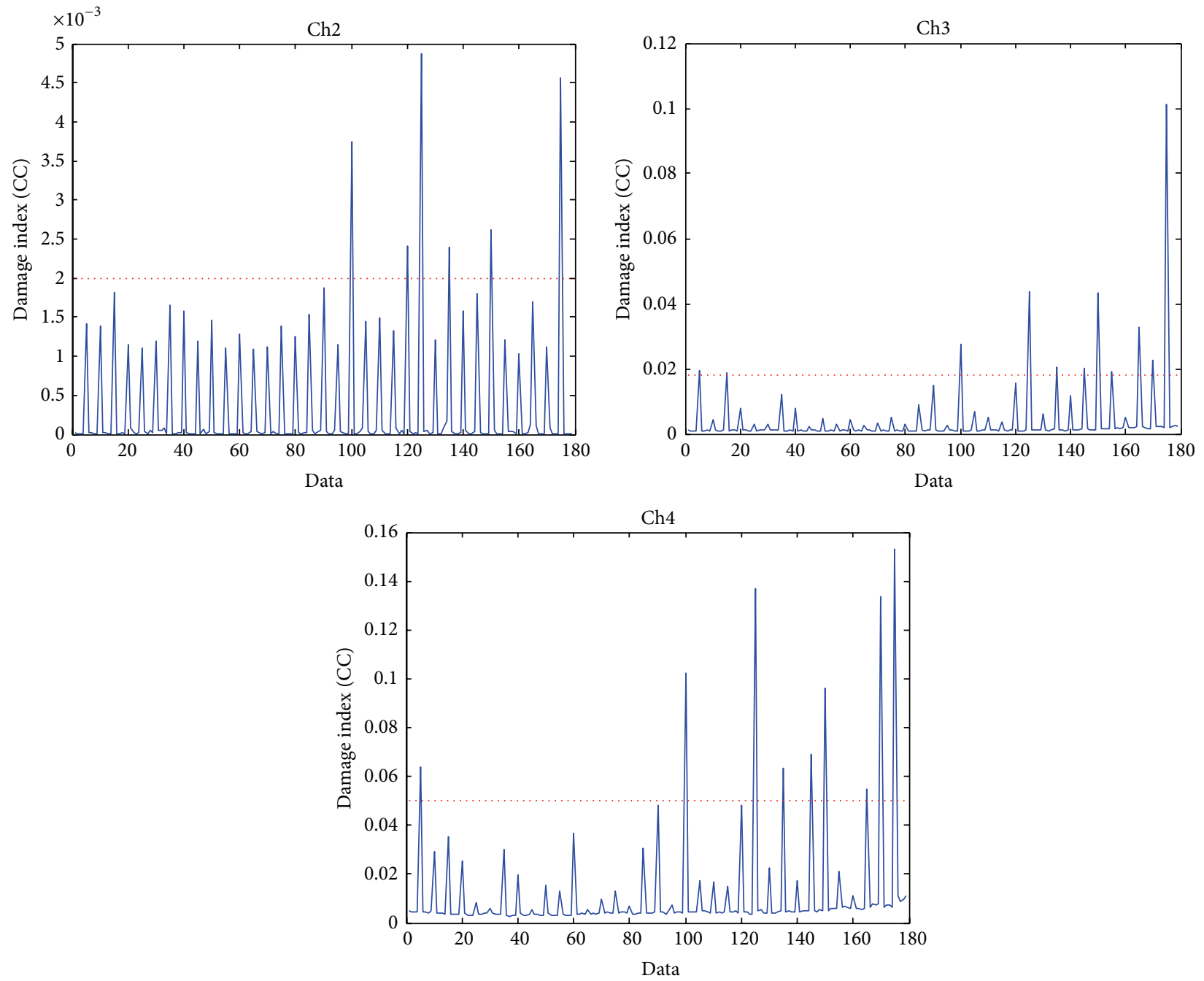

FIGURE 9: FRF-based DI values for Ch2-4. DI is measured between the current measurement and the previous reading. 

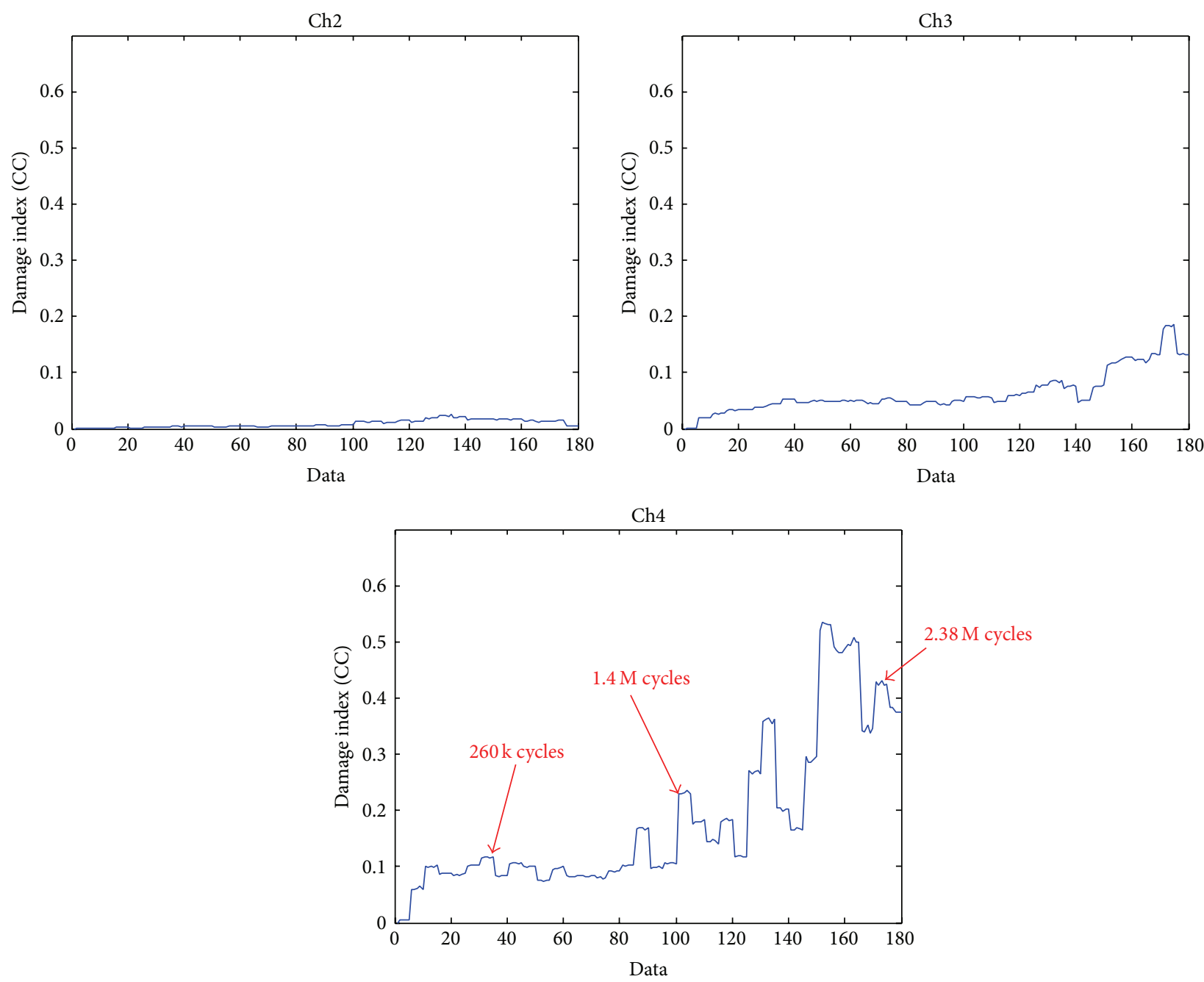

FIGURE 10: FRF-based DI values which are compared to a baseline for Ch2-4. DI is obtained between the current measurement and the baselines.

test setup is shown in Figure 7. The 9 meter blade was instrumented with eleven $2.5 \times 3.75 \mathrm{~cm}$ MFC sensors and one $5 \times 10 \mathrm{~cm}$ MFC actuator. The location of the sensors and the actuator in relation to the blade geometry is also shown in Figure 7. The blade underwent fatigue excitation at $2 \mathrm{~Hz}$ for defined intervals, and active-sensing data were collected between sessions while the fatigue excitation source was shut down. These data were collected from the eleven sensing channels at a sampling rate of $60 \mathrm{kHz}$, producing 32768 time points for each channel. A 7.5 RMS random excitation signal amplified by a factor of 30 was provided to the actuator. During the test, the fatigue damage was visually identified in the root area after 2.3 million cycles.

The collected data were converted to the frequency domain using FFT for the start of the fatigue cycling and at each cessation thereafter. The FRF obtained from the blade in the pristine condition was used to predict the system response from data collected at testing interval. FRF measurements of channels 3 and 4 are shown in Figure 8, for the frequency range of $1-25 \mathrm{kHz}$. Although extensive averaging was required to enhance the signal to noise ratio, the identified FRFs are repeatable and represent the dynamic characteristic of the local area of the structure.

The correlation coefficient between the current measurement and the previous reading was used as a feature to track the progression of structural change over the course of the fatigue test. In this way, the effect of fatigue loading given within the measurement interval could be estimated. The values of the correlation coefficients calculated at periodic intervals throughout the course of the test are shown in Figure 9. Note that plots are not in the same scale.

Right after starting the fatigue load, Ch3 and Ch4 showed some "settling" effects, which exhibit increases in DI values. All three results indicate that there is a large increase in data measured at data 100. This corresponds to the measurement made right after 1.43 million cycles. Although we did not have any reference data to compare against, the results indicate that there was likely a notable change in the blades integrity on the test just before this measurement was taken. In addition, the measurement made at 125 shows another substantial increase, 
which was made after 1.8 million cycles. Another change was observed in the data 150 , which was measured at 2.11 million cycles. Finally, the measurements taken on the final day (175 at 2.53 million cycles), all three measurements showed a large increase in the damage metrics, which indicates the on-set of structural damage on the blade. It is also interesting to note that the Ch4 (close to the damaged area) already showed certain increase in the DI value (170:2.38 million cycles) just before the final test, which may indicate the imminent damage.

Figure 10 depicts another FRF-based DI value. In this case, the measurements were compared to the baseline, which was measured on the first day of the test. The changes in FRF indicate changes in the structural parameters, which in turn indicate the presence of damage. By comparing the measurements made on the first day, these figures give relative changes in structural integrity. It can be seen that the values in Ch4 are substantially higher than Ch3 and Ch2, which indicates the damage is located close to this sensor.

The active-sensing technique at this fatigue test provides relatively repeatable responses, could detect damage, has localized sensing capability, and is less sensitive to operational variations. The method and analysis are relatively straightforward and do not require significant memory and power usage of the system, and the whole process can be embedded in the turbine blades with the appropriate development of hardware. It must be noted, however, that more than four sensors were broken during the test, as the adhesive was not ideal for a fatigue test. In addition, the hardware connectors did not function multiple occasions during the test. The sensor diagnostic procedure, which confirms the operational status of piezoelectric transducers $[17,18]$, should be implemented for fatigue test. Also, with the reduced number of sensors and a shorter traveling distance, the wave propagation technique was not considered in this test. These issues will be addressed for future tests.

\section{Summary}

This study investigated two piezoelectric active-sensing SHM techniques, including Lamb wave propagations and frequency responses, for wind turbine blade monitoring. With these techniques, the condition of the turbine blade can be qualitatively assessed with the presence and location of damage successfully identified using each method. In addition, the use of higher excitation frequencies enabled each method to be sensitive to small defects in the structure, while remaining insensitive to the effects of boundary and ambient condition changes on the response. This method can provide real-time health monitoring because the hardware and signal processing requirements can be significantly relaxed, especially in the case of FRF-based methods. This paper also summarizes the SHM results of a full-scale fatigue test of a $9 \mathrm{~m} \mathrm{CX}-100$ blade using piezoelectric active sensors, which confirms the localization capability of the active-sensing technique. The fatigue test also points out the importance of ensuring the robustness of sensing equipment and the reliability of the SHM system for field deployment under harsh operational conditions. This subject is currently being investigated by the authors.

\section{Conflict of Interests}

The authors declare that there is no conflict of interests regarding the publication of this paper.

\section{Acknowledgments}

This work was funded by the Department of Energy through the Laboratory Directed Research and Development Program at Los Alamos National Laboratory. This research was also partially supported by the Leading Foreign Research Institute Recruitment Program through the National Research Foundation of Korea funded by the Ministry of Education, Science and Technology (2011-0030065). G. Park acknowledges the partial support of the "Leaders Industry-University Cooperation" Project, supported by the Ministry of Education, Science and Technology (MEST), Republic of Korea. The authors also would like to acknowledge Mark Rumsey and Jon White from Sandia National Laboratory for their support and guidance on this study.

\section{References}

[1] U.S. Department of Energy, 20\% Wind Energy by 2030: Increasing Wind Energy's Contribution to U.S. Electricity Supply, Wind \& Hydropower Technologies Program, 2008, http://wwwl.eere.energy.gov/windandhydro/pdfs/41869.pdf.

[2] C. C. Ciang, J. R. Lee, and H. J. Bang, "Structural health monitoring for a wind turbine system: a review of damage detection methods," Measurement Science and Technology, vol. 19, no. 12, Article ID 122001, 2008.

[3] B. Lu, Y. Li, X. Wu, and Z. Yang, "A review of recent advances in wind turbine condition monitoring and fault diagnosis," in Proceedings of the IEEE Power Electronics and Machines in Wind Applications (PEMWA '09), pp. 1-7, Lincoln, Neb, USA, June 2009.

[4] N. Bourasseau, E. Moulin, C. Delebarre, and P. Bonniau, "Radome health monitoring with Lamb waves: experimental approach," NDT and E International, vol. 33, no. 6, pp. 393-400, 2000.

[5] S. S. Kessler, S. M. Spearing, and C. Soutis, "Damage detection in composite materials using Lamb wave methods," Smart Materials and Structures, vol. 11, no. 2, pp. 269-278, 2002.

[6] J. B. Ihn and F. K. Chang, "Detection and monitoring of hidden fatigue crack growth using a built-in piezoelectric sensor/actuator network: II. Validation using riveted joints and repair patches," Smart Materials and Structures, vol. 13, no. 3, pp. 621-630, 2004.

[7] V. Giurgiutiu, A. Zagrai, and J. J. Bao, "Piezoelectric wafer embedded active sensors for aging aircraft structural health monitoring," Structural Health Monitoring, vol. 1, no. 1, pp. 4161, 2002.

[8] M. Lemistre and D. Balageas, "Structural health monitoring system based on diffracted Lamb wave analysis by multiresolution processing," Smart Materials and Structures, vol. 10, no. 3, pp. 504-511, 2001. 
[9] A. Raghavan and C. E. S. Cesnik, "Review of guided-wave structural health monitoring," Shock and Vibration Digest, vol. 39, no. 2, pp. 91-114, 2007.

[10] G. Park, A. C. Rutherford, J. R. Wait, B. Nadler, C. R. Farrar, and T. N. Claytor, "High-frequency response functions for composite plate monitoring with ultrasonic validation," AIAA Journal, vol. 43, no. 11, pp. 2431-2437, 2005.

[11] S. H. Diaz Valdes and C. Soutis, "Delamination detection in composite laminates from variations of their modal characteristics," Journal of Sound and Vibration, vol. 228, no. 1, pp. 1-9, 1999.

[12] H. Zhang, M. J. Schulz, A. Naser, F. Ferguson, and P. F. Pai, "Structural health monitoring using transmittance functions," Mechanical Systems and Signal Processing, vol. 13, no. 5, pp. 765787, 1999.

[13] J. P. Dunne, D. M. Pitt, K. J. Kilian, and D. A. Sofge, "Recent advances in active damage interrogation," in Proceedings of the 42nd AIAA/ASME/ASCE/AHS/ASC Structures, Structural Dynamics, and Materials Conference and Exhibit Technical Papers, pp. 1937-1945, Seattle, Wash, USA, April 2001.

[14] G. Park, H. Sohn, C. R. Farrar, and D. J. Inman, "Overview of piezoelectric impedance-based health monitoring and path forward," Shock and Vibration Digest, vol. 35, no. 6, pp. 451-463, 2003.

[15] D. Berry, Design of 9-Meter Carbon-Fiberglass Prototype Blades: CX-100 and TX-100, NM SAND2007-0201, Sandia National Laboratories, Albuquerque, NM, USA, 2007.

[16] V. Giurgiutiu, "Tuned Lamb wave excitation and detection with piezoelectric wafer active sensors for structural health monitoring," Journal of Intelligent Material Systems and Structures, vol. 16, no. 4, pp. 291-305, 2005.

[17] G. Park, C. R. Farrar, A. C. Rutherford, and A. N. Robertson, "Piezoelectric active sensor self-diagnostics using electrical admittance measurements," Journal of Vibration and Acoustics, vol. 128, no. 4, pp. 469-476, 2006.

[18] G. Park, C. R. Farrar, F. L. di Scalea, and S. Coccia, "Performance assessment and validation of piezoelectric active-sensors in structural health monitoring," Smart Materials and Structures, vol. 15, no. 6, pp. 1673-1683, 2006. 

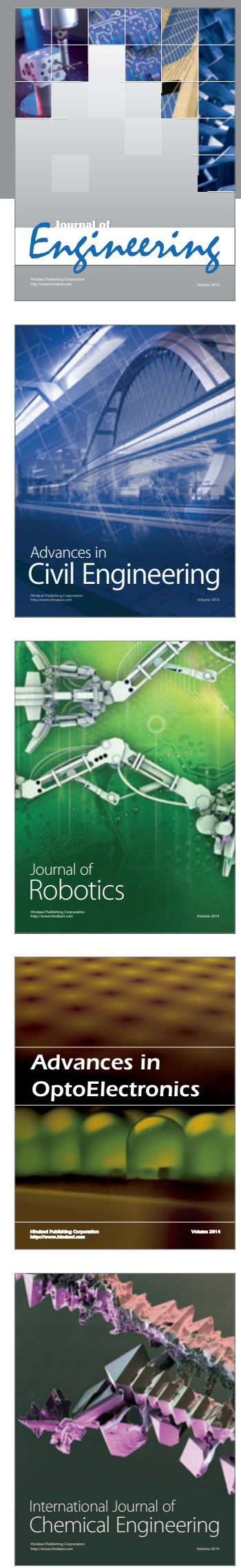

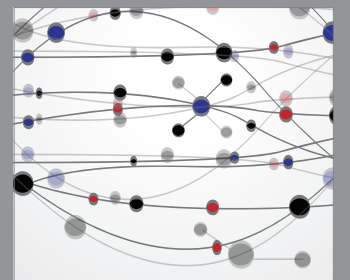

The Scientific World Journal
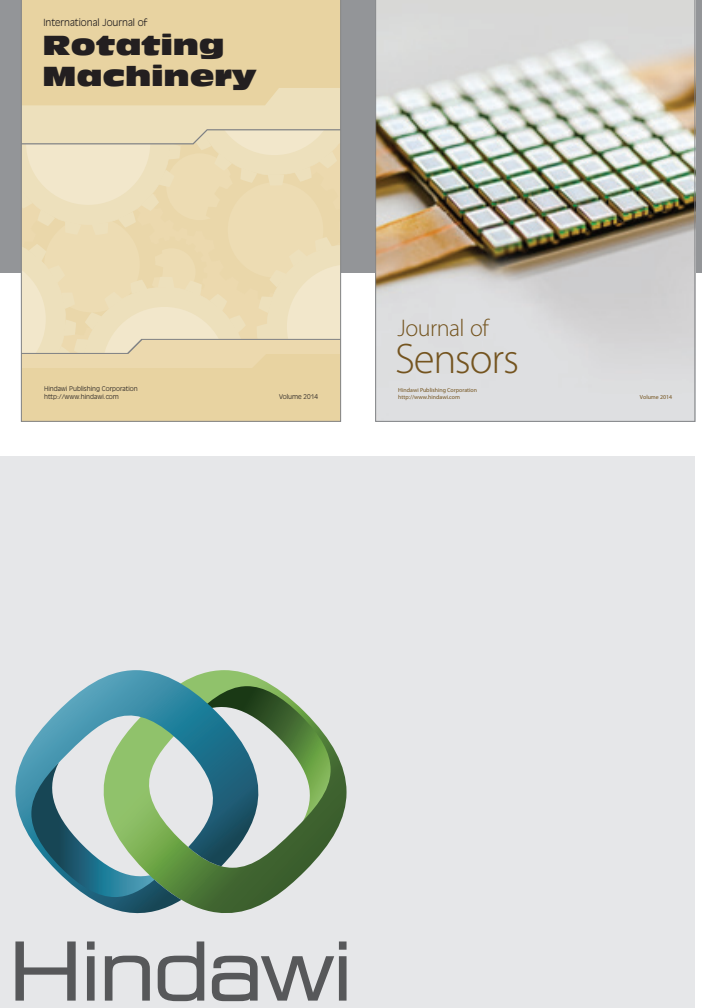

Submit your manuscripts at http://www.hindawi.com
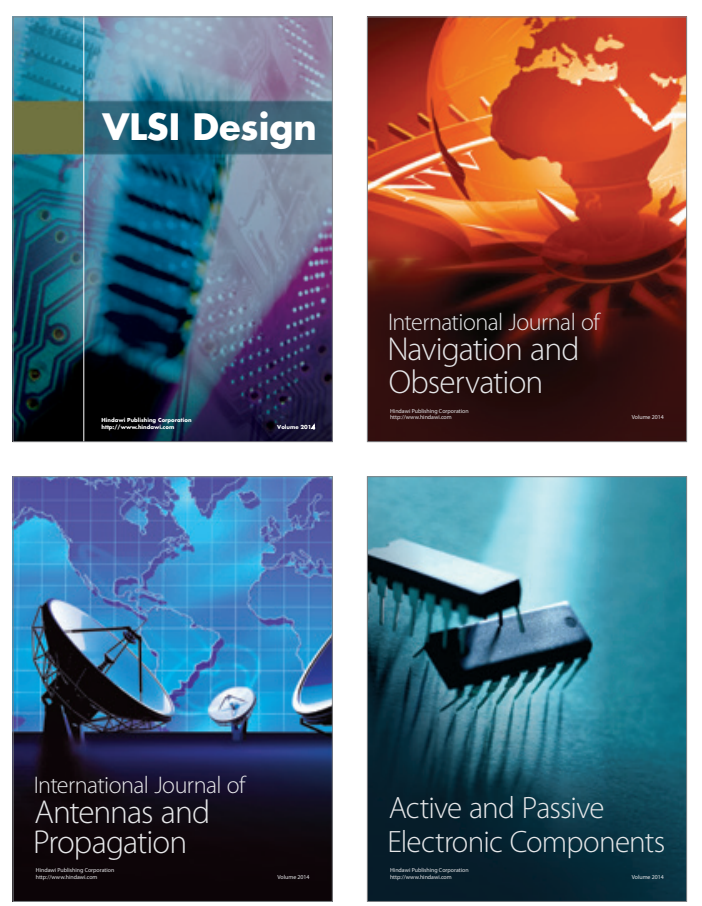
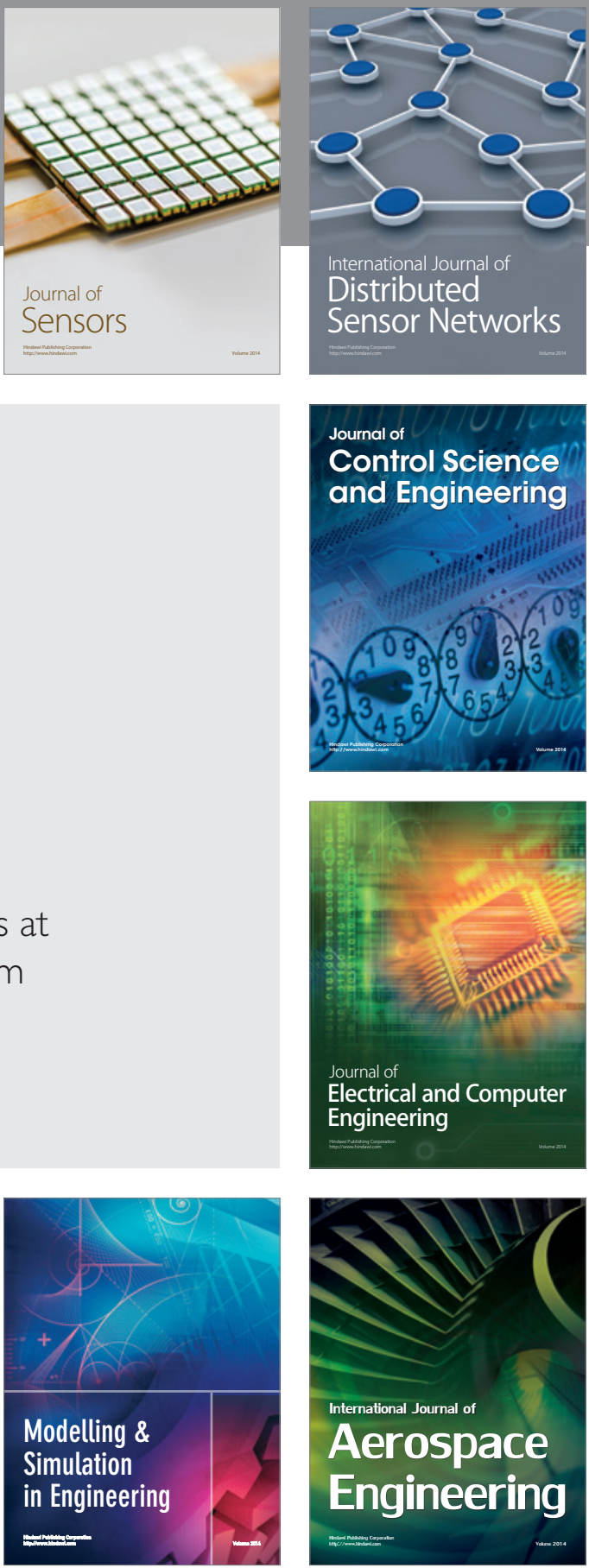

Journal of

Control Science

and Engineering
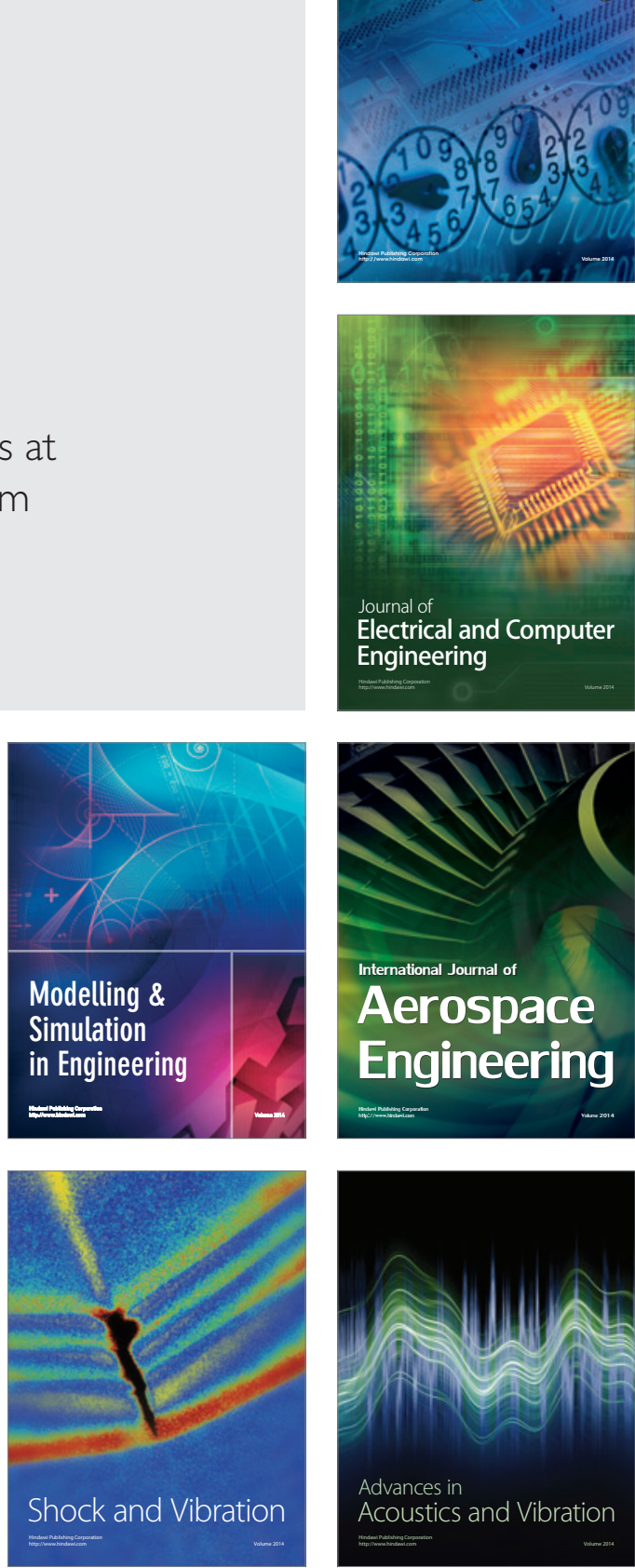